\title{
Review of long-term adverse effects associated with the use of chemically-modified animal and nonanimal source hyaluronic acid dermal fillers
}

\author{
Paul C Edwards' \\ John E Fantasia ${ }^{2}$
}

'Associate Professor (Clinical), Department of Periodontics and Oral Medicine, Pathology and Oncology, University of Michigan School of Dentistry, Ann Arbor MI, USA;

${ }^{2}$ Chief, Division of Oral Pathology, Department of Dental Medicine, Long Island Jewish Medical Center, New Hyde Park, NY, USA
Correspondence: Paul C Edwards Department of Periodontics and Oral Medicine, Pathology and Oncology, University of Michigan School of Dentistry, I0I I North University Avenue, Ann Arbor, MI 48I09-1078, USA Email pedwards@dentalpathologist.com, paulce@umich.edu

\begin{abstract}
Although only recently introduced, chemically-modified hyaluronic acid dermal fillers have gained widespread acceptance as "redefining" dermal fillers in the fields of dermatology and cosmetic facial surgery. Although hyaluronic acid-based dermal fillers have a low overall incidence of long term side effects, occasional adverse outcomes, ranging from chronic lymphoplasmacytic inflammatory reactions to classic foreign body-type granulomatous reactions have been documented. These long-term adverse events are reviewed.
\end{abstract}

Keywords: hyaluronic acid, Restylane ${ }^{\circledR}$, Hylaform ${ }^{\circledR}$, injectable dermal filler, foreign body reaction, granuloma

\section{Introduction}

A major goal of cosmetic surgeons, dermatologists and pharmaceutical companies has been the development of biocompatible materials with prolonged clinical longevity for use as esthetic facial soft tissue augmentation agents. Ideal properties of soft tissue fillers include biocompatibility (low risk of foreign body-type reaction), reasonable clinical appearance and duration, ease of use and minimal tendency to migrate to distant sites.

Currently available materials can be broadly subcategorized as nonbiodegradable (permanent) or biodegradable (temporary). The biodegradable materials can be further subdivided into those of intermediate or long duration. Examples of permanent materials include liquid silicone (eg, Silikon $\left.{ }^{\circledR}\right)$, solid silicon particles in suspension (eg, Bioplastique ${ }^{\circledR}$ ), polymethyl methacrylate microspheres with bovine collagen $\left(\mathrm{eg}\right.$, Artecoll $\left.^{\circledR}\right)$, acrylic hydrogel particles with unmodified hyaluronic acid (eg, Dermalive ${ }^{\circledR}$ ), calcium hydoxylapatite (eg, Radiesse ${ }^{\circledR}$, formerly called Radiance), and various polyacrylamide gel formulations (eg, Aquamid ${ }^{\circledR}$ ). Many of these permanent fillers are associated with a definite risk of delayed foreign body-type reactions (Vargas-Machuca et al 2006). Biodegradable materials include polylactic acid microspheres (eg, New-Fill ${ }^{\circledR}$ ), allogeneic human collagen from tissue culture (eg, CosmoDerm ${ }^{\circledR}$ ), autologous fat and collagen grafts, and xenogeneic material such as bovine collagen (eg, Zyderm ${ }^{\circledR}$ ) and glutaraldehyde-treated bovine collagen (eg, Zyplast $\left.{ }^{\circledR}\right)$. Until recently, bovine collagen was regarded as the "gold standard" in facial soft tissue augmentation. However, the use of bovine collagen is associated with a 3\%-5\% risk of delayed hypersensitivity reactions (Lowe et al 2001), necessitating double skin testing prior to treatment (Narins et al 2003).

To date, no universally applicable dermal filler has been developed, although manufacturers of hyaluronic acid-based products claim that their products are close to fulfilling many of the requirements of an ideal tissue augmentation agent. Based on the rapid acceptance of these materials by both clinicians and patients, it would appear that 
many practitioners believe that there may be some validity to these claims. Hyaluronic acid-based temporary dermal fillers are being employed with increasing frequency for the treatment of facial skin lines and for lip augmentation procedures (Carruthers and Carruthers 2003). It is estimated that in the United States in 2004 alone, 878,000 patients were treated with hyaluronic acid-based fillers (Matarasso et al 2006), both animal source (Hylaform, Biomatrix Inc., Ridgefield, NJ; a hyaluronic acid extract derived from rooster combs) and nonanimal source (Restylane ${ }^{\circledR}$, Q-Medical Corporation, Uppsala, Sweden; a cross-linked hyaluronic acid injectable filler produced from bacteria by microbiologic engineering techniques).

As stated by Walker (2006), although the aging process itself has been extensive researched, "there is a paucity of data and peer-reviewed papers on human responses to interventions in aging," much of which involves "replacement therapy". This paper will review the potential long-term side effects associated with the use of cross-linked hyaluronic acid injectable fillers as replacement therapy in dermatology/ plastic surgery.

\section{Structure and biochemistry of hyaluronic acid}

Glycosaminoglycans (GAGs), also referred to as mucopolysaccharides, are large negatively-charged unbranched polymers composed of pairs of repeating sugar units, one of which is an amino sugar. The main GAGs include chondriotin-4-sulphate, chondroitin-6-sulfate, dermatan sulfate, heparan sulfate, keratan sulfate, heparin, and hyaluronic acid. Of these, hyaluronic acid (hyaluronan), consisting of repeating units of the monosaccharide D-glucuronic acid and the amino sugar N-acetyl-D-glucosamine linked together via alternating beta-1,4 and beta-1,3 glycosidic bonds, is the most prevalent. It has been estimated that the average $60 \mathrm{~kg}$ human body contains $12 \mathrm{~g}$ of hyaluronic acid (Matarasso et al 2006). Polymers of hyaluronan can range in size from 4,000 to $20,000,000$ Daltons in vivo.

\section{Synthesis and degradation of hyaluronic acid}

In the extracellular matrix (ECM), hyaluronic acid is produced predominantly by fibroblasts through a complex of cytoplasmic proteins on the plasma membrane called hyaluronan synthases and is extruded through the plasma membrane into the extracellular space. Hyaluronan is ultimately degraded in a step-wise manner by a family of hyaluronidase enzymes (Stern 2003). The first step in the process is the cleavage of high molecular weight hyaluronic acid to $20 \mathrm{kDa}$ fragments. These fragments are further degraded into smaller units, primarily tetrasaccharides. These different-sized hyaluronic acid molecules have varying biological effects.

\section{Function of hyaluronic acid}

$\mathrm{HA}$ is a major component of the ECM of the dermis, where it is a major contributor to the formation a resilient gel-like ground substance that resists compressive forces.

The traditional view of hyaluronic acid as simply being an inert "filler" material has recently been questioned (Stern 2003). Hyaluronic acid contributes to tissue hydrodynamics by creating space for the movement of cells. Hyaluronic acid is believed to regulate the diffusion of nutrients, metabolites, and hormones between cells, and stimulates fibroblast proliferation, migration and collagen production. Hyaluronic acid also regulates cell proliferation and motility by regulating cell/cell and cell/matrix interactions through the cell membrane receptor CD44 (Stern 2003).

The high molecular weight hyaluronic acid molecules have anti-inflammatory, anti-angiogenic, and immunosuppressive properties, whereas the $20 \mathrm{kDa}$ fragments and the very low molecular weight hyaluronic acid degradation products stimulate the synthesis of new blood vessels, inflammatory cytokines and induce inflammatory responses in macrophages and dendritic cells secondary to infection and tissue injury.

Hyaluronic acid is present in high concentrations in embryonic tissue and in malignant neoplasms. Overexpression of CD44 has been linked to the growth of a number of malignant neoplasms, and in some cancers, hyaluronan levels correlate with poor prognosis. Hyaluronic acid likely contributes to tumor growth via its interaction with the CD44 receptor (Hill et al 2006).

\section{Medical applications of hyaluronic acid}

The widespread tissue distribution of hyaluronic acid, being a major component of tissue ranging from skin and cartilage to vitreous humor, accounts in part for the potential of hyaluronic acid as a universal soft tissue replacement material. Another benefit of hyaluronic acid is that, in contrast to collagen, its chemical structure is reportedly identical across different species (Richter et al 1979). Therefore, the risk of imunogenicity to hyaluronic acid-derived products is believed to be low. In addition, as a result of its water-binding affinity, hyaluronic acid forms a high viscosity hydrated 
polymer that purportedly maintains much of its volume by binding additional molecules of water as it degrades.

The first hyaluronic acid-based biomedical product, Healon, was developed as an ophthalmic-surgical aid for use in various anterior segment procedures, as a vitreous replacement after vitrectomy and in retinal detachment surgery. Hyaluronic acid has also been used in the treatment of osteoarthritis of the knee as a joint fluid supplement, typically administered by injection into the knee joint.

The process of aging is characterized by loss of resiliency and atrophy of underlying adipose tissue. A progressive reduction in hyaluronic acid content has been described in aging skin (Ghersetich et al 1994), leading to the suggestion that variations of hyaluronic acid levels in the dermis may account for some of the changes seen in aged skin, including decreased turgidity, wrinkling, and decreased elasticity. In view of these observations and the fact that hyaluronic acid is a major contributor to the extracellular matrix of the dermis, hyaluronic acid-derived products have been extensively investigated as injectable dermal supplements. The principal drawback with purified hyaluronic acid dermal filler is the short half-life of hyaluronic acid in the dermis, estimated at 24-48 hours. In order to increase the longevity of hyaluronic acid to the point that it is practical for clinical use, pharmaceutical companies have developed longer lasting hyaluronic acid formulations through chemical cross-linking.

Hyaluronic acid-derived dermal fillers have been available in Europe since 1996 (Andre et al 2005). The first chemically-modified hyaluronic acid filler approved by the US Food and Drug Administration (FDA) for the correction of moderate to severe wrinkles and skin folds was Restylane, approved in December 2003. Chemically modified hyaluronic acid formulations currently available in the United States include the nonanimal source hyaluronic acid (NASHA) materials: Restylane, Restylane Fine Line, Restylane Perlane, Restylane SubQ, Juvederm (Inamed Corporation, Santa Barbara, California) and Captique (Inamed Corporation, Santa Barbara, California). The FDA has also approved a line of animal-derived hyaluronic acid products: Hylaform Regular, Hylaform Fine and Hylaform Plus. Differences between these formulations include hyaluronic acid source, particle size, degree of cross-linking, and concentration of hyaluronic acid. Hylaform is cross-linked by glutaraldehyde vinyl sulfone, whereas Restylane is stabilized by treatment with 1,4-butandiol diglycidylether. The concentration of hyaluronic acid in Restylane is approximately four-fold that in Hylaform; $20 \mathrm{mg} / \mathrm{ml}$ versus $5.5 \mathrm{mg} / \mathrm{ml}$ (Patel et al 2006).
In addition to the correction of moderate to severe wrinkles and skin folds, hyaluronic acid-based dermal fillers are also widely used for the correction of scars and for lip augmentation. Intradermal injection of hyaluronic acid is contraindicated in patients with autoimmune disorders, on immunosuppressive therapy, in patients with active herpetic lesions, and in patients with acneiform lesions.

Because of their limited half-life, most patients require at least twice yearly injections of chemically-modified hyaluronic acid products. Some (Hamra 2006) have argued that, from a long-term economic perspective, patients may be better served by conventional plastic surgery in certain clinical scenarios.

\section{Adverse reactions to hyaluronic acid-derived dermal fillers}

Adverse reactions to any type of surgical intervention/ replacement therapy can be divided into immediate-to-short term, those occurring within the first 1-2 weeks, and long-term, those occurring several weeks to months after the original intervention. Short duration local injection site reactions, generally resulting from an acute inflammatory response to tissue damage combined with the introduction of a foreign material, include swelling, pain, tenderness and bruising, and are seen in the vast majority of patients treated with injectable hyaluronic acid derivatives (Narins et al 2003). In general, lip augmentation is associated with the highest incidence of post-injection pain, swelling, and bruising. In addition, recurrent herpes labialis occurs in a percentage of patients following lip augmentation with NASHA. Consequently, many clinicians prescribe oral prednisone and/or systemic antiviral agents to reduce the severity of swelling and to reduce the incidence of developing recurrent herpetic lesions when performing lip injections.

Although much less common, more serious immediate onset reactions have included the development of injection site ulcers following inadvertent arterial occlusion (eg, Schanz et al 2002; Lowe 2003; Hirsch et al 2007), especially when injecting in the glabellar area. A protocol has been suggested to manage patients who develop signs indicative of impending vascular occlusion (Glaich et al 2006). Visual disturbances have also been described following retinal branch artery occlusion secondary to hyaluronic acid injection (Peter and Mennel 2006). One case of angioedema-like swelling of the lip following injection of Restylane was documented (Leonhardt et al 2005), although others have suggested that this phenomenon is very common following 
injection of Restylane into the lips and does not represent a true angioedema reaction (Klein 2005).

Late infections following hyaluronic acid injection are rare. Toy and Frank (2003) described two women in New York who developed granulomatous infections following injections of a non-FDA approved hyaluronic acid derivative (Hyacell) imported from South America. Both women were injected by the same practitioner, who was practicing medicine without a license. This practitioner was ultimately tied to a cluster of Mycobacterium abscessus infections, presumably related to injections with tainted hyaluronic acid.

The overall incidence of long term adverse reactions secondary to the dermal injection of hyaluronic acid skin fillers is believed to be low, the vast majority representing a foreign body-related chronic inflammatory reaction.

In a randomized, double-blinded study (Narins et al 2003) in which 138 patients received both Restylane and glutaraldehyde-cross linked bovine collagen (Zyplast, McGhan Medical Inc., Santa Barbara, California) on contralateral nasolabial folds, the frequency, intensity and duration of injection site reactions were similar for both products. Delayed onset reactions, primarily consisting of localized skin redness which resolved without treatment within 2-3 months, were noted in $8 \%$ of patients treated with Restylane.

A review (Friedman et al 2002) of all reports of adverse reactions to Restylane submitted to the manufacturer in 1999 and 2000, representing an estimated 306,000 treated patients, documented one adverse event for every 650 to 1800 patients treated. These reactions consisted primarily of localized hypersensitivity reactions. There were two cases of injection site necrosis in the glabellar area, as well as "rare reports of localized granulomatous reactions, bacterial infections, acneiform and cystic lesions". The exact number and type of "adverse reactions" was not fully detailed. The authors acknowledged that: (i) the number of total patients treated may have been overestimated, as the number was projected based on the total number of syringes of Restylane sold during this time period; and (ii) the total number of adverse reactions may have been under-reported by clinicians.

A retrospective study (Andre 2004) of an estimated 4300 patients treated with Restylane documented an overall incidence of hypersensitivity of $0.8 \%$, almost equally divided between immediate and delayed reactions. It was noted that the incidence of adverse reactions decreased to approximately $0.6 \%$ from 2000 to 2001 . The authors attributed this decrease to a reduction in the amount of protein in the raw product secondary to improvements in the manufacturing process. Overall, 18 delayed reactions, appearing between several weeks to 6 months after treatment, were documented. Most presented as nodules with associated erythema of the overlying skin. Three "sterile abscesses" were noted.

In a prospective randomized study of 38 individuals treated with Restylane alone or Restylane with Botulinum toxin A (Carruthers and Carruthers 2003), one subject in the combined Restylane/Botulinum toxin A group developed delayed erythema, swelling and discomfort at the injection site at 4 and 11 months.

A review of 709 patients (Lowe et al 2001) treated with either Hylaform or Restylane for smoothing of nasolabial and glabellar lines, lip augmentation and correction of atrophic facial scars, demonstrated an incidence of delayed skin reactions of slightly less than $0.5 \%$. These reactions, first noticed between 6 to 8 weeks after injection, were of varying severity and manifested as injection site induration, erythema and tenderness. One patient developed "a sterile abscess" of the nasolabial region. Time to complete resolution varied from 6 to 24 weeks. Three patients were treated with intralesional injections of triamcinolone. Subsequent intradermal skin testing for hyaluronic acid was positive in 4 of 5 patients tested.

Most examples of adverse reactions following dermal infiltration with hyaluronic acid-based fillers have been in the form of individual case reports (eg, Jordan 2005). Lupton and Alster (Lupton and Alster 2000) reported on a woman who developed multiple erythematous papulocystic nodules following injection of an unspecified "modified hyaluronic acid gel". The patient declined a biopsy. Another patient was described (Shafir et al 2000) who developed multiple "sterile abscesses" following injection of her lips and nasolabial folds with Restylane. Two of these lesions ruptured spontaneously, whereas the remainder required surgical excision. A similar case was described (Wolfram et al 2006) of another patient who developed multiple foreign body reactions following treatment with Restylane that also required surgical intervention to adequately manage. Patel and colleagues (2006) presented the case of a 65-year old woman who developed a hypersensitivity reaction following at least four previous treatments with Restylane. The reaction was characterized by severe injection site erythema, edema and induration. Interestingly, this patient had undergone a single negative intradermal Restylane challenge test in the forearm prior to undergoing the cosmetic procedures. The authors 
acknowledged that, in retrospect, the patient should have undergone a conventional double skin test.

\section{Histologic examination of dermally implanted hyaluronic acid}

In an in vivo study in rats (Fernandez-Cossio and CastanoOreja 2006), subcutaneous injection of Restylane was characterized by an acute inflammatory response within the first 4 weeks. At 8 months, there was little evidence of biodegradation. The implanted hyaluronic acid remained walled off from the surrounding tissue by a capsule consisting of collagen and fibroblasts. Minimal infiltration of cells into the implant was noted and there was no connective tissue formation within the implant. Inflammation was minimal. An in vivo rabbit histologic study of a new modified hyaluronic acid filler, in which hyaluronic acid molecules were cross-linked by an esterification reaction without introduction of another chemical agent (Alessandrini et al 2006), demonstrated intense macrophage-mediated phagocytosis of the injected filler. No evidence of persistent inflammation or granulomatous reaction was noted. One intradermal animal study (Sasaki et al 2003) with hylan (Synvisc), a chemically-modified hyaluronic acid injectable that is widely used to treat knee pain associated with degenerative osteoarthritis, resulted in severe foreign body-type granulomatous inflammation in guinea pigs. Although antibodies against hylan were described, no evidence of an antibody reaction directed against pure, unmodified hyaluronic acid was noted.

Few studies have documented histologic evidence of the in vivo longevity or adverse reactions to chemically-modified hyaluronic acid dermal injections in human subjects. One of the most comprehensive histopathologic assessments of adverse reactions following injection of cosmetic fillers was reported by Lombardi and colleagues (2004). Biopsies obtained from patients injected with Dermalive, which consists of $60 \%$ pure hyaluronic acid and $40 \%$ acrylic hydrogel, revealed classic foreign body giant cell-type granulomas. However, none of the patients biopsied in this study had been treated with hyaluronic acid-derived fillers alone.

Lamperle and colleagues (2003) examined tissue from several different injectable fillers, including Restylane, following intradermal infiltration into the volar forearm. Time periods ranged from 1-9 months. The authors concluded that although all substances tested appeared to be clinically safe, all exhibited some degree of undesirable side effects. In another study (Parada et al 2005), histologic specimens were obtained following adverse reactions to 6 different dermal fillers, including Restylane.

Most reports have described the presence of foreign body-type granulomatous reactions, although injection site reactions characterized by lymphoplasmacytic chronic inflammatory-type reactions have also been described (eg, Lowe et al 2001). Micheels (2001) documented 8 cases of delayed reactions to both Restylane and Hylaform in 219 patients treated with one or both of these products. These patients subsequently underwent intradermal testing with both Hylaform and Restylane preparations that had been pretreated with hyaluronidase. Biopsy results were reported from either the dermal testing site or the original facial site of injection on 4 of these patients. Findings ranged from classic foreign body-type giant cell granulomas (3 Hylaform, 1 Restylane) to chronic inflammation (1 Restylane). Although Micheels's reference to "anti-hyaluronic acid antibodies" has been widely reported in a number of other manuscripts, these findings have been criticized by others (Klein 2005) for several reasons, including that fact that the specificity of these antibodies to HA was not confirmed and the observation that hyaluronidase-treated preparations were used to elicit the reaction instead of unmodified filler.

Fernandez-Acenero and colleagues (2003) reported a 48-year old woman who developed a nodule of the upper lip 2 months after receiving a single injection of Restylane. On biopsy, this was characterized as a foreign body-type giant cell granulomatous reaction surrounding a pool of amorphous material consistent with unresorbed HA.

Raulin and colleagues (2000) performed skin testing on a 53 year-old woman who developed a granulomatous-like lesion after injection of Hylaform for correction of perioral wrinkles. A biopsy taken from the cutaneous test site after 30 days revealed the presence of a foreign body granuloma as well as the presence of mucin-like basophilic material presumed to be Hyalform. In a similar case (Rongioletti et al 2003), biopsy on a 72-year-old woman who developed induration of the skin after receiving injections of Restylane revealed a foreign body granulomatous reaction encircling clear spaces of variable size. Alcian blue positive material was identified in the sclerotic stroma, but not in the clear vacuolated areas. Due to the histologic resemblance of the biopsied specimen to a silicone foreign body reaction, infrared spectrophotometry was performed to rule out the possibility of adulteration of the injected filler with silicone. This case was unusual because, during the same period, the patient was diagnosed with scleromyxedema, a rare connective tissue disorder of unknown etiology characterized by 


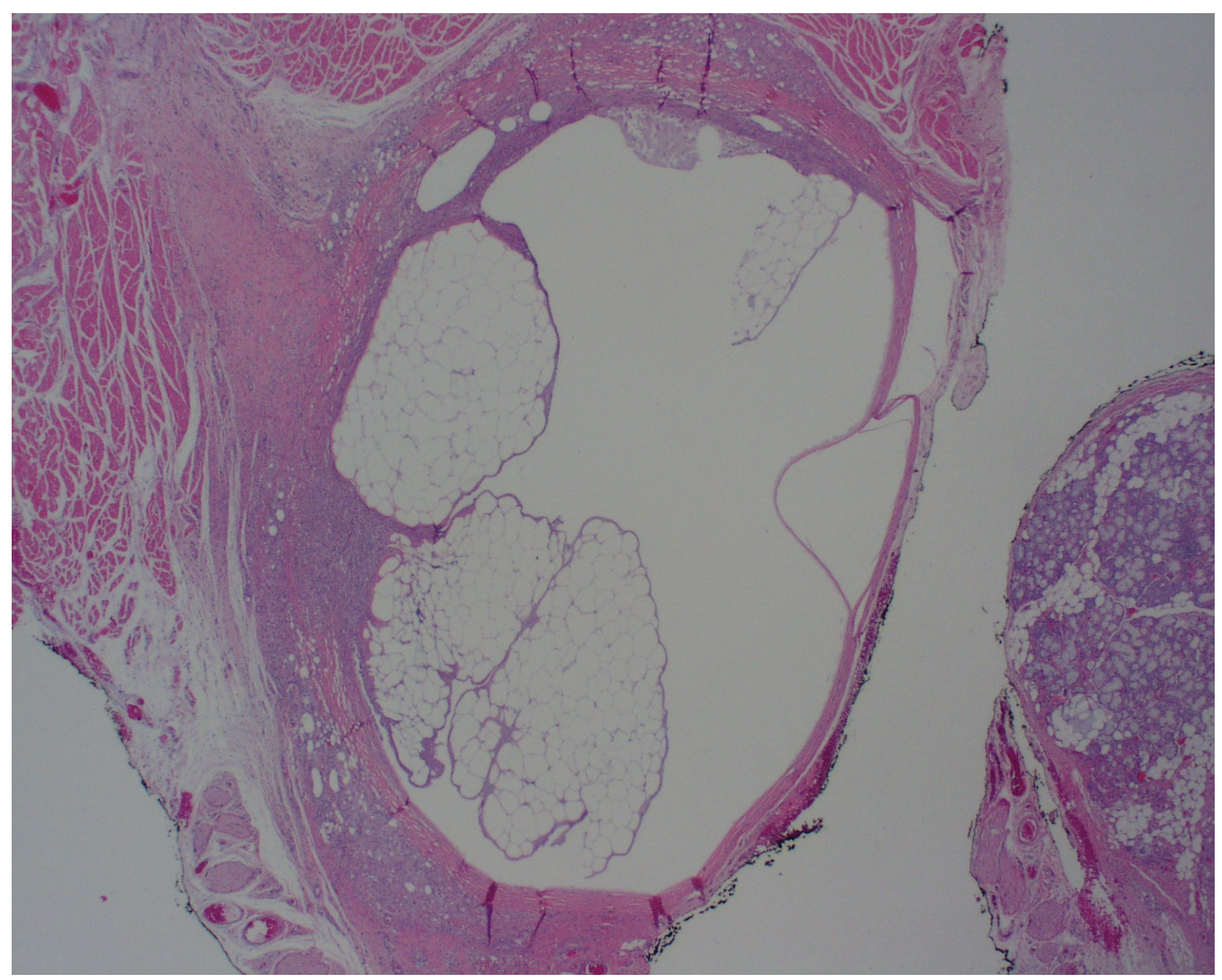

Figure I Low power view of biopsy specimen from lower lip from a 74-year-old woman who presented with a submucosal nodule of the lower lip. The patient had received injections of Restylane to the lips approximately 6 months before. Histologic examination of the biopsied specimen revealed the presence of multiple vacuolated, cyst-like areas. These are surrounded by a fibrotic connective tissue capsule and a tissue reaction composed predominantly of histiocytes and foamy macrophages. (Hematoxylin and eosin, original magnification 10x).

the presence of excessive hyaluronic acid deposition in the dermis coupled with the presence of abnormal paraprotein. A direct association between these two findings could not be confirmed.

Other examples (Honig et al 2003) have included a woman who developed a foreign body-type giant cell reaction following injections of hyaluronic acid to the nasolabial fold, and a 41 year old woman who developed multiple indurated, erythematous injection site nodules 5 weeks after receiving her first treatment with Restylane in the nasolabial, glabellar and periorbital areas (Ghislanzoni et al 2006). Histologic examination in the latter case revealed the presence of honeycomb-shaped basophilic material, presumed to represent hyaluronic acid gel, surrounded by a foreign body giant cell-type granulomatous reaction.
Dal Sacco and colleagues (2005) described a patient who developed multiple tender nodules in the nasolabial fold 4 months after injection of Restylane. Biopsy revealed the presence of sarcoid-like granulomas. Interestingly, the patient also developed similar-appearing dermal lesions at previous sites of venipuncture and scarring. On further diagnostic workup, the patient was diagnosed with sarcoidosis. Based on the extent of her clinical disease and the presentation of dermal lesions in multiple sites of previous skin trauma, it was concluded that the hyaluronic acid injections triggered a sarcoidal skin reaction in a background of a pre-existing but subclinical case of sarcoidosis ("scar sarcoidosis").

We previously reported (Edwards et al 2006) a case of a 74-year-old woman who presented with a $1.5 \mathrm{~cm}$, firm, submucosal nodule of the lower lip that was clinically 


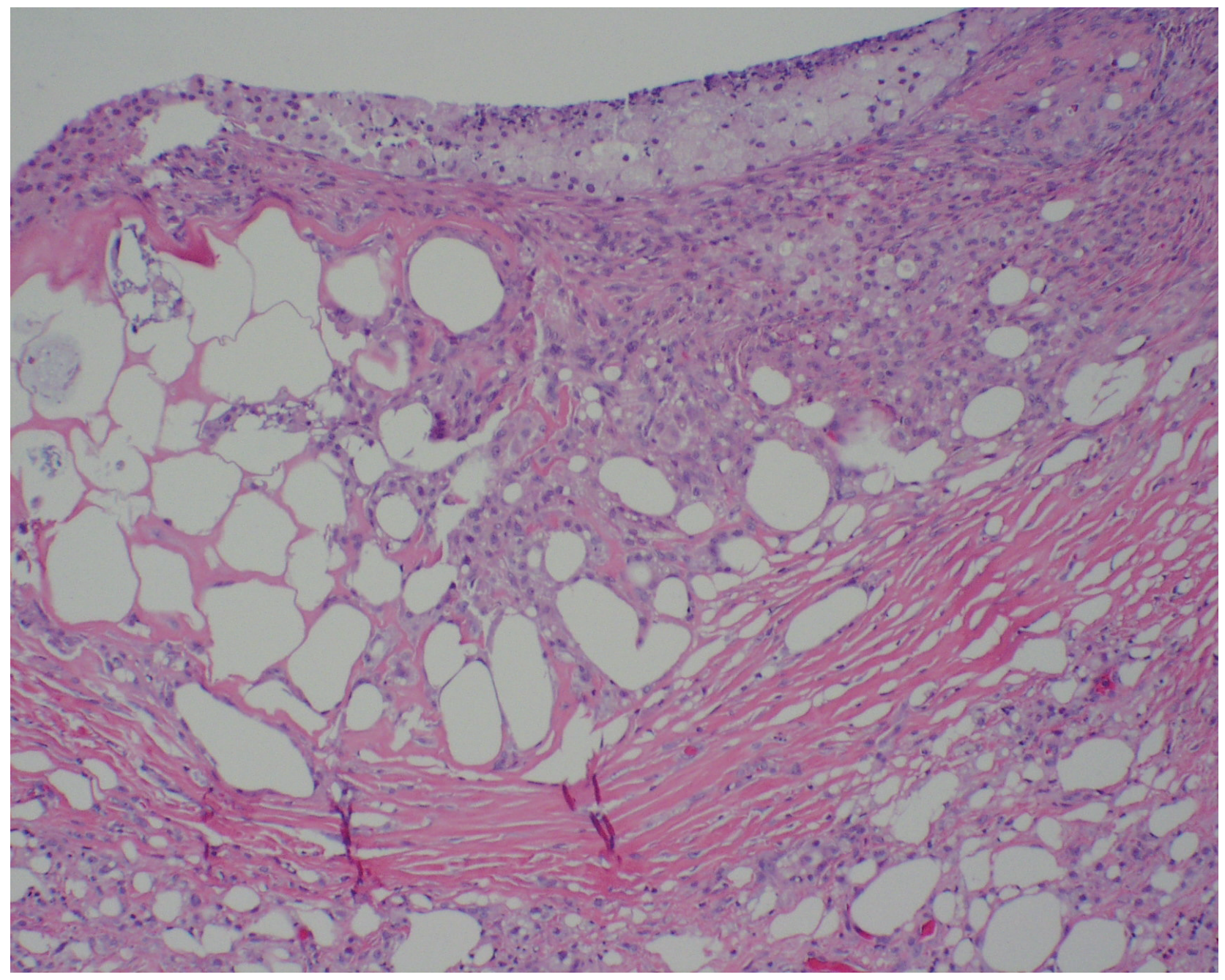

Figure 2 High power view of biopsy specimen from lower lip. Fibrotic tissue and abundant histiocytes surround the vacuolated areas. (Hematoxylin and eosin, original magnification 40x).

thought to represent a benign minor salivary gland neoplasm. Histologic examination (Figures 1 and 2) of the biopsied specimen revealed the presence of multiple vacuolated cyst-like areas, surrounded by a granulomatous foreign body reaction composed predominantly of histiocytes, foamy macrophages and lymphocytes. Multinucleated giant cells were also noted (Figure 3). On subsequent questioning, the patient acknowledged that she had received injections of Restylane to the lips by a dermatologist approximately 6 months before the development of the nodule, but had failed to mention this because she didn't think that these two events were related. The patient denied having any additional cosmetic procedures to the face or lips other than the Restylane injection.

Although the clinical longevity of hyaluronic acid-derived products appears to be in the range of 4 to 6 months in most dermal applications, there is evidence that the tissue longevity of these products may be substantially longer. In one case (Bennet and Taher 2005), the continued presence of Restylane was documented in the dermis of the lip 23 months post-implantation. Soparkar and colleagues (2004) performed an incisional biopsy on a 65 -year-old female patient who developed multiple plaque-like elevations of the periorbital region 5 years after undergoing cosmetic treatment with Restylane. Histologic examination revealed the presence of multiple cyst-like spaces, some of which contained alcian blue positive material, with a surrounding fibrotic reaction. Interestingly, these dermal elevations disappeared following the local infiltration of hyaluronidase, suggesting that at least in some cases the presence of excessive volumes of injected hyaluronic acid may play a role in the development of these late reactions.

Very little has been published regarding the more recently introduced NASHA materials Captique and Juvederm. There is one case in the literature (Matarasso and Herwick 2006) 


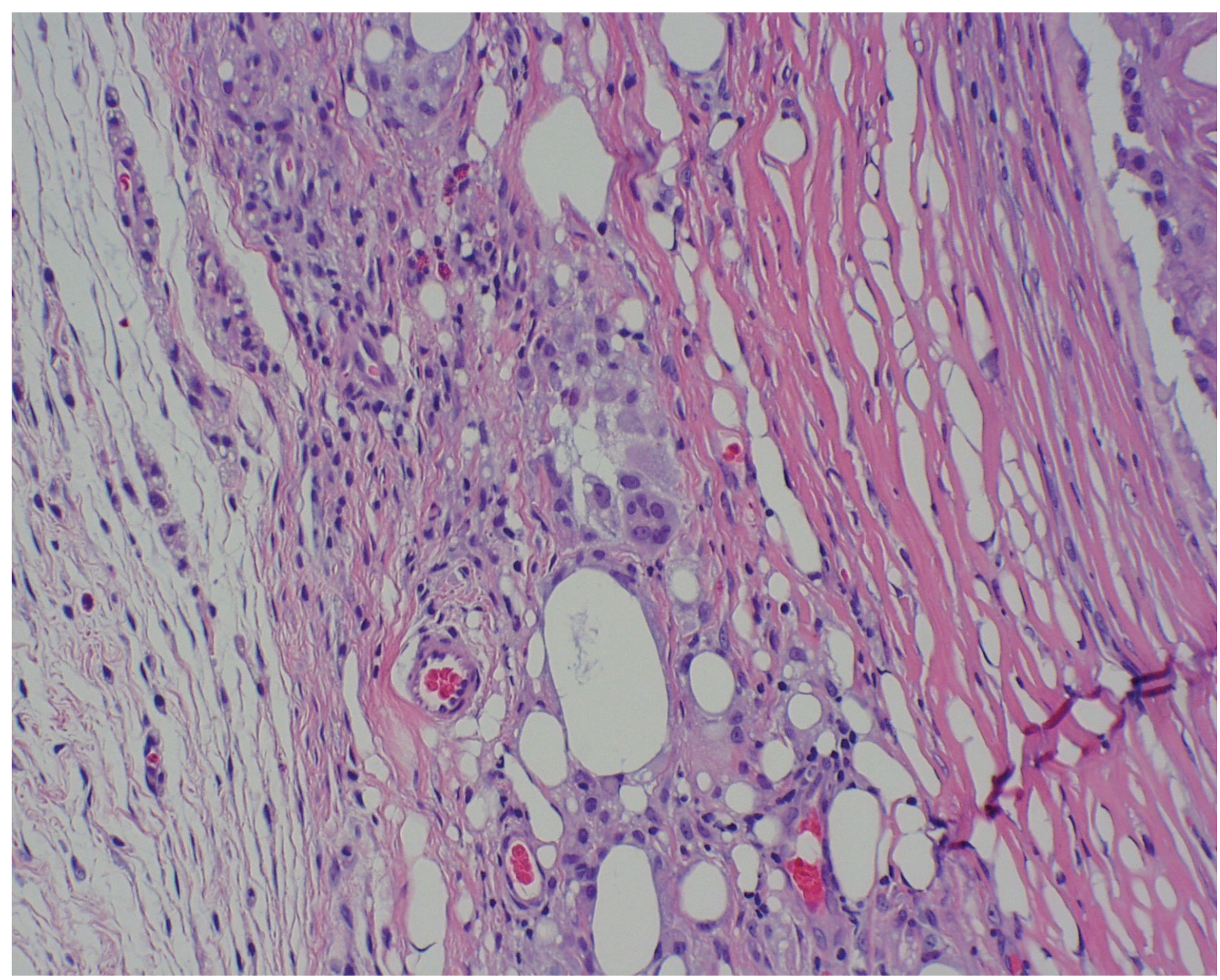

Figure 3 High power view of biopsy specimen from lower lip. A multinucleated foreign body-type giant cell is visible in the center of the photomicrograph. (Hematoxylin and eosin, original magnification 60x).

of a 52-year old woman who developed biopsy-confirmed foreign body injection site granulomas several weeks after injections of Captique to the nasolabial folds and perioral area. These resolved spontaneously after 6 months.

\section{Treatment of hyaluronic acid-related late reactions}

Treatment options for patients presenting with delayed hypersensitivity reactions range from simple observation to local treatment with topical corticosteroids or intralesional injection of corticosteroids. Lesions that fail to resolve with conservative therapy may benefit from treatment with systemic therapy with corticosteroids, although in some case surgical intervention may ultimately be required. Intralesional administration of hyaluronidase has also been successfully employed to resolve nodular lesions (Soparkar and Patrinely 2005). Recently, etanercept, an inhibitor of tumor necrosis factor-alpha activity, has been used to resolve lesions in patients with silicone granulomas (Desai et al 2006), although its use in treating patients presenting with delayed hypersensitivity reactions to hyaluronic acid has not been documented to date.

\section{Pathogenesis of foreign body reactions to hyaluronic acid-based dermal fillers}

Injection of any foreign material into the dermis triggers an inflammatory response; essentially a protective response intended to eliminate the initial cause of cell injury as well as to repair any tissue damaged as a result of the original insult. The initial phase, lasting from several hours to days, is characterized by an acute inflammatory process localized to the injection site. The hallmark of acute inflammation involves circulatory changes leading to the release of soluble mediators of inflammation, and chemotaxis of neutrophils to the site of injury. If these areas of acute inflammation fail to 
resolve and become walled off, abscess formation ensues. By definition, an abscess is defined as an acute inflammatory process characterized by a localized collection of dead and dying neutrophils surrounding a foreign agent or organism.

Long term tissue reactions to any unresorbed foreign material are generally characterized by a chronic inflammatory reaction, consisting of an infiltration of mononuclear cells (eg, macrophages, lymphocytes and plasma cells) and attempts at healing characterized by the formation of a collagen capsule and/or granulation tissue. Many lateoccurring adverse reactions to injectable dermal agents most likely represent localized foreign body-type granulomatous inflammation. Granulomatous inflammation represents a specific type of chronic inflammation caused by antigens that evoke cell-mediated hypersensitivity (eg, tuberculosis) or agents that persist at site of inflammation (eg, foreign bodies). The histologic presentation of granulomatous inflammation is composed of modified macrophages, termed epithelioid macrophages, which can occasionally fuse to form "foreign body-type" multinucleated giant cells, as well as T-lymphocytes, occasional plasma cells, and a proliferation of fibroblasts and capillaries. The epithelioid macrophages are typically found adherent to the implant surface, contributing to the formation of a soft tissue capsule.

In the dermatologic literature, the term "sterile abscess" is broadly used to describe a nodular lesion that has formed as a result of foreign bodies and/or injected medications that have not been totally absorbed. In many cases, these "sterile abscesses" represent a chronic inflammatory granulomatous reaction. From a cosmetic point of view, surgical treatment to evacuate the contents of the "sterile abscess" may be required to reduce the likelihood of indurated scar-like tissue forming (Lowe et al 2005).

The exact cause of these chronic inflammatory reactions following the injection of chemically-modified hyaluronic acid fillers has been debated. The literature in this area makes extensive reference to the fact that, because hyaluronic acid is identical across species, these products are not recognized as foreign by the body and therefore should not trigger any long-term inflammatory response. However, as reviewed in this manuscript, it is apparent that although the risk of developing a clinically evident host reaction is limited, numerous cases have nevertheless been documented. The most widely accepted view is that these late side effects are related to contamination by residual bacterial and or avian proteins from the production process. These products do indeed contain trace amounts of hyaluronin-associated protein and, in the case of Restylane, streptococcus equi-derived bacterial antigens (Andre 2004). The documented decrease in the incidence of late adverse reactions following improvements in the Restylane manufacturing process, resulting in a substantial decrease in protein contaminants, has been widely cited in support of this theory (Friedman et al 2002).

Another possible explanation may lie in the chemical cross-linking process that is used to increase the clinical half-life of these products compared to that of native hyaluronic acid. While remnants of the chemical agents used in the cross-linking ("stabilizing") process could trigger a delayed inflammatory reaction, it is more likely that the chemical cross-linking process itself, which reportedly modifies the structure by $0.6 \%-1.0 \%$, (Andre 2004), introduces an immunogenic potential by inducing changes in the three dimensional structure of these molecules. The breakdown products of these chemically-modified hyaluronic acids could also be a cause of the immunologic response. Alternatively, these breakdown products could themselves be further metabolized to other immunogenic compounds (Coleman 2005).

The possibility that some of these long-term side effects could be in part technique-related, for example related to local deposition of excessive tissue volumes of hyaluronic acid, cannot be entirely discounted.

Of interest is the observation that, in vivo, hyaluronic acid is depolymerized into lower molecular weight fragments by enzymatic digestion. Even more intriguing are recent findings that hyaluronic acid and its degradation products are important regulators of dendritic cells and macrophages (Termeer et al 2003). Hyaluronic acid degradation products are potent activators of macrophages through the CD44 cell surface receptors (Leonhardt et al 2005), while dendritic antigen-presenting cells and T-cells appear to regulate the synthesis and degradation of hyaluronic acid directly by synthesizing hyaluronic acid synthetases and hyaluronidases. Although only speculation, it is plausible that changes in the balance between high molecular weight hyaluronic acid and its degradation products in a local microenvironment, induced by the injection of chemicallymodified hyaluronic acid particles (with altered degradation kinetics) could lead to altered T-cell and/or macrophage activation and hence granuloma formation in a certain subset of patients.

\section{Conclusions}

Although chemically-modified hyaluronic acid dermal fillers, both animal and nonanimal source, have a very low 
incidence of long term side effects, patients need to be informed of the potential risk of foreign body reactions to these injectable agents.

\section{References}

Alessandrini A, Di Bartolo C, Pavesio A, et al. 2006. ACP gel: a new hyaluronic acid-based injectable for facial rejuvination. Preclinical data in a rabbit model. Plast Reconstr Surg, 118:341-6.

Andre P. 2004. Evaluation of the safety of a non-animal stabilized hyaluronic acid (NASHA- Q-Medical, Sweden) in European countries: a retrospective study from 1997 to 2001. J Eur Acad Dermatol Venereol, $18: 422-5$.

Andre P, Lowe NJ, Parc A, et al. 2005. Adverse reactions to dermal fillers: a review of European experiences. J Cosmetic Laser Surg, 7:171-6.

Bennet R, Taher M. 2005. Restylane persistent for 23 months found during Mohs micrographic surgery: a source of confusion with hyaluronic acid surrounding basal cell carcinoma. Dermatol Surg, $31: 1366-9$

Carruthers J, Carruthers A. 2003. A prospective, randomized, parallel group study analyzing the effect of BTX-A (Botox) and nonanimal sourced hyaluronic acid (NASHA, Restylane) in combination compared with NASHA (Restylane) alone in severe glabellar rhytides in adult female subjects: treatment of severe glabellar rhytides with a hyaluronic acid derivative compared with the derivative and BTX-A. Dermatol Surg, 29:802-9.

Coleman SR. 2005. Cross-linked hyaluronic acid fillers. Plastic Reconstr Surg, 117:661-5.

Dal Sacco D, Cozzani E, Parodi A, et al. 2005. Scar sarcoidosis after hyaluronic acid injection. Int $J$ Dermatol, 44:411-12.

Desai AM, Browning J, Rosen T. 2006. Etanercept therapy for silicone granuloma. J Drugs Dermatol, 5:894-6.

Edwards PC, Fantasia JE, Iovino R. 2006. Foreign body reaction to hyaluronic acid (Restylane): an adverse outcome of lip augmentation. J Oral Maxillofac Surg, 64:1296-9.

Fernandez-Acenero MJ, Zamora E, et al. 2003. Granulomatous foreign body reaction against hyaluronic acid: report of a case after lip augmentation. Dermatol Surg, 29:1225-6.

Fernandez-Cossio S, Castano-Oreja MT. 2006. Biocompatibility of two novel dermal fillers: histological evaluation of implants of a hyaluronic acid filler and a polyacrylamide filler. Plast Reconstr Surg, 117:1789-96

Friedman PM, Mafong EA, Kauvar AN, et al. 2002. Safety data of injectable nonanimal stabilized hyaluronic acid gel for soft tissue augmentation. Dermatol Surg, 28:491-4.

Ghersetich I, Lotti T, Campanile G, et al. 1994. Hyaluronic acid in cutaneous intrinsic aging. Int J Dermatol, 33:119-22.

Ghislanzoni M, Bianchi F, Barbareschi M, et al. 2006. Cutaneous granulomatous reaction to injectable hyaluronic acid gel. Brit J Dermatol, 154:755-8.

Glaich AS, Cohen JL, Goldberg LH. 2006. Injection necrosis of the glabella: protocol for prevention and treatment after use of dermal fillers. Dermatol Surg, 32:276-81.

Hamra ST. 2006. \$25,000 and still counting. Plastic Reconstructive Surg, 118:801-3.

Hill A, McFarlane S, Johnston PG, et al. 2006. The emerging role of CD44 in regulating skeletal micrometastasis. Cancer Letts, 237:1-9.

Hirsch RJ, Lupo M, Cohen JL, et al. 2007. Delayed presentation of impending necrosis following soft tissue augmentation with hyaluronic acid and successful management with hyaluronidase. J Drugs Dermatol, 6:325-8.

Honig JF, Brink U, Korabiowska M. 2003. Severe granulomatous allergic tissue reaction after hyaluronic acid injection in the treatment of facial lines and its surgical correction. J Craniofac Surg, 14:197-200.

Jordan JR. 2005. Delayed inflammatory reaction to hyaluronic acid (Restylane). Ophthal Plast Reconstr Surg, 21:401-2.
Klein AW. 2005. Re: hypersensitivity reactions to injectable hyaluronic acid: reality or fiction. Dermatol Surg, 31:1745.

Lemperle G, Morhenn V, Charrier U. 2003. Human histology and persistence of various injectable filler substances for soft tissue augmentation. Aesthetic Plast Surg, 27:354-66.

Leonhardt JM, Lawrence N, Narins RS. 2005. Angioedema acute hypersensitivity reaction to injectable hyaluronic acid. Dermatol Surg, 31:577-9.

Lombardi T, Samson J, Plantier F, et al. 2004. Orofacial granulomas after injection of cosmetic fillers. Histopathologic and clinical study of 11 cases. J Oral Pathol Med, 33:115-20.

Lowe NJ. 2003. Re: arterial embolization caused by injection of hyaluronic acid (Restylane). Brit J Dermatol, 148:379-80.

Lowe NJ, Maxwell CA, Lowe PL, et al. 2001. Hyaluronic acid skin fillers: adverse reactions and skin testing. J Am Acad Dermatol, 45:930-3.

Lowe NJ, Maxwell CA, Patnaik R. 2005. Adverse reactions to dermal fillers: review. Dermatol Surg, 31:1616-25.

Lupton JR, Alster TS. 2000. Cutaneous hypersensitivity reaction to injectable hyaluronic acid gel. Dermatol Surg, 26:135-7.

Matarasso SL, Carruthers JD, Jewell ML, et al. 2006. Consensus recommendations for soft-tissue augmentation with nonanimal stabilized hyaluronic acid (Restylane). Plastic Reconstructive Surg, $117:$ s3-34.

Matarasso SL, Herwick R. 2006. Hypersensitivity reaction to nonanimal stabilized hyaluronic acid. J Am Acad Dermatol, 55:128-31.

Micheels P. 2001. Human anti-hyaluronic acid antibodies: is it possible? Dermatol Surg, 27:185-91.

Narins RS, Brandt F, Leyden J, et al. 2003. A randomized, double-blind, multicenter comparison of the efficacy and tolerability of Restylane versus Zyplast for the correction of nasolabial folds. Dermatol Surg, 29:588-95.

Parada MB, Michalany NS, Hassun KM, et al. 2005. A histologic study of adverse effects of different cosmetic skin fillers. Skinmed, 4:345-9.

Patel VJ, Bruck MC, Katz BE. 2006. Hypersensitivity reaction to hyaluronic acid with negative skin testing. Plast Reconstr Surg, 117:e92-4.

Peter S, Mennel S. 2006. Retinal branch artery occlusion following injection of hyaluronic acid (Restylane). Clin Experiment Ophthalmol, 34:363-4.

Raulin C, Greve B, Hartschuh W, et al. 2000. Exudative granulomatous reaction to hyaluronic acid (Hylaform). Contact Dermatitis, 43:178-9.

Richter AW, Ryde EM, Zetterstrom EO. 1979. Nonimmunogenicity of a purified sodium hyaluronate preparation in man. Int Arch Allergy Appl Immunol, 59:45-8.

Rongioletti F, Cattarini G, Sottofattori E, et al. 2003. Granulomatous reaction after intradermal injections of hyaluronic acid gel. Arch Dermatol, 139:815-16.

Sasaki M, Miyazaki Y, Takahashi T. 2003. Hylan G-F 20 induces delayed foreign body inflammation in guinea pigs and rabbits. Toxicol Pathol, 31:321-5.

Schanz S, Schippert W, Ulmer A, et al. 2002. Arterial embolization caused by injection of hyaluronic acid (Restylane). Brit J Dermatol, 146:920-32.

Shafir R, Amir A, Gur E. 2000. Long-term complications of facial injections with Restylane (injectable hyaluronic acid). Plast Reconst Surg, 106:121-6.

Soparkar CN, Patrinely JR. 2005. Managing inflammatory reaction to Restylane. Ophthalmic Plastic Reconstr Surg, 21:151-64.

Soparkar CN, Patrinely JR, Tschen J. 2004. Erasing Restylane. Ophthal Plast Reconstr Surg, 20:317-18.

Stern R. 2003. Devising a pathway for hyaluronan catabolism: are we there yet? Glycobiology, 13:r105-15.

Termeer C, Sleeman JP, Simon JC. 2003. Hyaluronan-magic glue for the regulation of the immune response? Trends Immunology, $24: 112-14$ 
Toy BR, Frank PJ. 2003. Outbreak of Mycobacterium abscessus infection after soft tissue augmentation. Dermatol Surg, 29:971-3.

Vargas-Machuca I, Gonzalez-Guerra E, Angulo J, et al. 2006. Facial granulomas secondary to Dermalive microimplants: report of a case with histopathologic differential diagnosis among the granlomas secondary to different injectable permanent filler materials. Am J Dermatopathol, 28:173-7.

Walker RF. 2006. On the evolution of anti-aging medicine. Clinical Interventions in Aging, 1:201-3.

Wolfram D, Tzankov A, Piza-Katzer H. 2006. Surgery for foreign body reactions due to injectable fillers. Dermatology, 213:300-4. 
